\title{
A "Clinical" Trial of Two Marking Protocols for Multiple Choice Assessments
}

Authors: Jill Barber and Douglas Steinke. Manchester Pharmacy School, The University of Manchester

Background: Multiple choice questions are a staple of university, school and professional examinations. A problem with these assessments, however, is that a student who knows nothing and guesses the answer to every question achieves a raw mark of $100 / \mathrm{n} \%$ where $\mathrm{n}$ is the number of options per question. So in an examination where the questions have five possible responses, students are gifted $20 \%$. Two common protocols exist to ensure that student who knows nothing gets $0 \%$, and the student who knows everything gets $100 \%$. In traditional negatively marked MCQs a correct answer attracts 1 mark, an incorrect answer $-1 /(n-1)$ and a unanswered question 0 . Alternatively, a "guessing correction" or normalisation is applied; this is mathematically equivalent to a correct answer attracting 1 mark, an incorrect answer $-1 /(n-1)$ and a null answer also $-1 /(n-1)$. The second protocol is thus mathematically less generous than the first but anecdotal evidence suggests that it is less unpopular.

Description of work : We will investigate the hypothesis that students are so riskaverse that they score less well on the mathematically more generous protocol. This is a cross-over trial, to be conducted during a first year assessment taken by all students. Students will be divided into two groups of equal ability and simultaneously tested on 10 questions, each with five possible answers. Group A will be told that they are marked by traditional MCQs; group B by the use of a guessing correction. Students will then be tested on a further 10 questions with the protocols reversed. For an $80 \%$ power with $5 \%$ representing a significant result, we require 16 questions and 100 students to achieve $95 \%$ confidence.

Proposed evaluation: Quantitative data will be collected as described above and qualitative data assessing students' perceptions of the two protocols will also be gathered and analysed. 